\title{
Influência da temperatura e substrato na germinação de sementes de jabuticabeira (Myrciaria cauliflora Berg.) ${ }^{1}$
}

\author{
Influence of temperature and substrate on the germination of jaboticaba \\ (Myrciaria cauliflora Berg.)
}

\author{
Maristela Aparecida Dias²; José Carlos Lopes ${ }^{3}$; José Dias de Souza Neto ${ }^{4}$ : Elaine Heberle ${ }^{2}$
}

\begin{abstract}
RESUMO
O trabalho foi desenvolvido no Laboratório de Análise de Sementes da Universidade Federal do Espírito Santo, em Alegre- ES. O objetivo foi avaliar o efeito de diferentes temperaturas e substratos sobre a germinação e vigor de sementes de jabuticabeira. Para isso se utilizou como substrato areia pura (A), terra+areia+esterco bovino (T+A+E), pó de casca de coco (PC) e Hortimix ${ }^{\circledR}$ $(\mathrm{H})$, e temperaturas constantes de 20 , e $30^{\circ} \mathrm{C}$ e alternada de $20-30^{\circ} \mathrm{C}$. O delineamento experimental foi inteiramente casualizado, com quatro repetições. As médias de germinação, índice de velocidade de emergência, plantas normais, massa fresca e massa seca de plantas foram comparadas entre si pelo teste de Tukey a 5\%. A temperatura de $30^{\circ} \mathrm{C}$ e o substrato composto por terra, areia e esterco bovino em iguais proporções foram mais favoráveis a germinação da espécie nas condições avaliadas.
\end{abstract}

Palavras-chave: Myrciaria cauliflora, germinação, vigor.

\begin{abstract}
The experiment was developed in the Seeds Analysis Laboratory in the University of Espirito Santo, Alegre, Brazil. The objective was to study the effect of different temperatures and substrates on the germination and strength of Jaboticaba tree seeds. The substrates used were pure sand $(A)$, soil + sand + cow dung $(T+A+E)$, coconut rind powder $(P C)$, Hortimix ${ }^{\circledR}(H)$; temperature treatments were $20^{\circ} \mathrm{C}, 30^{\circ} \mathrm{C}$ and alternation between these two temperatures. The experimental design was completely random, with four repetitions. The germination average, emergence rate, plant fresh weight and dry weight were compared using the Tukey's test at $5 \%$. The temperature of $30^{\circ} C$ and the soil + sand + cow dung $(T+A+E)$ substrate in equal proportions was the most efficient combination of the conditions studied for the germination of this species.
\end{abstract}

Key words: Myrciaria cauliflora, germination, vigor.

\section{Introdução}

A jabuticabeira se destaca entre as espécies cultivadas em pomares domiciliares no Brasil, sendo amplamente utilizada tanto para o consumo ao natural, quanto na fabricação de geléias, vinhos e licores caseiros. Pertence à família Myrtaceae, uma das mais importantes famílias frutíferas de ocorrência no Brasil, da qual fazem parte a goiaba, araçá e pitanga, dentre outras. Originária do centrosul brasileiro, a espécie pode ser encontrada desde
Minas Gerais até o Rio Grande do Sul. A espécie em estudo, Myrciaria cauliflora Berg. apresenta de duas a três floradas ao ano. A polpa do fruto é suculenta, esbranquiçada, de sabor doce e levemente ácido, na qual se encontram mergulhadas de uma a quatro sementes. As sementes apresentam viabilidade curta e emergência lenta, devendo ser semeadas em até cinco dias após a colheita. Apesar de sua origem subtropical, pode adaptar-se a vários climas, se desenvolvendo melhor nos climas mesotérmicos dos planaltos do sudeste, podendo tolerar

Projeto desenvolvido pelo Programa Voluntário de Iniciação Científica (PIVIC/UFES), ano de 2005.

Engenheira agrônoma (UFES), mestranda em Fitotecnia (UFV), Brasil. E-mail: diasmunizf@gmail.com

Engenheiro agrônomo, Ds. em Fitotecnia, Prof. Adj. Departamento Produção Vegetal - UFES, Brasil.

Estudante do curso de Agronomia. Universidade Federal Espírito Santo-UFES, Brasil. 
geadas de curta duração. Em relação aos solos, desenvolve-se bem em solos diversos, preferindo os silico-argilosos (Gomes, 1987; Donadio, 2000; Emater, 2007; Danner et al., 2007).

Temperatura e substrato são fatores importantes para o desenvolvimento vegetal. No processo de germinação, o substrato atua como suporte onde se condicionam as sementes para germinar, fornecendo condições adequadas para o desenvolvimento do processo e para o crescimento das plântulas (Figliola et al., 1993). Normalmente os substratos comercializados apresentam características físico-químicas adequadas à formação inicial de diversas espécies, porém o alto custo pode inviabilizar sua utilização por parte do produtor. Dessa forma, a escolha do substrato deve ser feita considerando a facilidade na sua obtenção, formas de preparo, formulação e custo do mesmo (Danner et al., 2007). Poucas informações são disponíveis a cerca da influência do substrato utilizado sobre a germinação e vigor de sementes de Jabuticabeira, algumas delas são fornecidas por Alexandre et al. (2004) e Alexandre et al. (2010) no qual se compararam os substratos areia e vermiculita e substrato comercial.

Com relação à temperatura, as sementes apresentam comportamento variável, pois não há temperatura ótima e uniforme definida para a germinação para todas as espécies. De forma geral, a temperatura máxima para germinação de muitas sementes, encontra-se entre 35 e $40^{\circ} \mathrm{C}$ e a ótima, entre 15 e $30^{\circ} \mathrm{C}$. Entretanto, temperaturas situadas na faixa de 20 a $30^{\circ} \mathrm{C}$ têm se mostrado adequadas à germinação das espécies tropicais e subtropicais (Albrecht et al., 1986; Marcos-Filho, 1986; Borges e Rena, 1993).

Apesar do avanço no processo de propagação assexuada, a propagação via sementes ainda é a técnica mais utilizada para a jabuticabeira, devido às dificuldades encontradas no enraizamento dessa espécie (Manica, 2000). De acordo com Danner et al. (2007) o uso de sementes é importante no processo de obtenção de mudas para porta-enxertos, no melhoramento genético e na manutenção da variabilidade.

Devido à escassez de estudos sobre o comportamento das sementes de jabuticabeira com relação à fisiologia da germinação e vigor, este trabalho teve o objetivo de avaliar o efeito de diferentes temperaturas e substratos sobre o comportamento germinativo das sementes de jabuticabeira.

\section{Material e Métodos}

O trabalho foi desenvolvido no Laboratório de Tecnologia e Análise de Sementes do Centro de Ciências Agrárias da Universidade Federal do Espírito Santo (CCA/UFES), Alegre-ES. Foram utilizadas sementes de frutos de jabuticabeira coletados em pomares domiciliares da região.

As sementes foram extraídas manualmente dos frutos recém colhidos e a polpa removida através de lavagem em água corrente. Para remoção da mucilagem, utilizou-se garrafa plástica de dois litros, areia e solução de hipoclorito $2 \%$, na qual as sementes foram agitadas manualmente durante cinco minutos. Posteriormente, as sementes foram cuidadosamente lavadas em água corrente e dispostas sobre papel absorvente para secagem à sombra por 24 horas. Foi avaliado o comportamento de embebição das sementes, utilizando-se quatro repetições de 25 sementes. As pesagens foram realizadas a cada hora durante as oito primeiras horas e após 24, 48 e 72 horas de embebição. Os resultados foram dados em porcentagem do aumento de peso em relação ao peso da massa seca das sementes.

Para realização dos testes de germinação e vigor, foram utilizadas quatro repetições de 50 sementes, tratadas com solução de BENLAT ${ }^{\circledR}$ (BENOMYL 500) na concentração de $750 \mathrm{mg} \mathrm{L}^{-1}$ por três minutos, semeadas nos diferentes substratos umedecidos de acordo com as Regras para Análise de Sementes (Mapa, 1992). Os substratos testados foram areia pura (A), terra+areia+esterco bovino $(\mathrm{T}+\mathrm{A}+\mathrm{E})$, pó de casca de coco (PC) e Hortimix ${ }^{\circledR}$ $(\mathrm{H})$, submetidos às temperaturas constantes de $20,30{ }^{\circ} \mathrm{C}$ e alternada de $20-30{ }^{\circ} \mathrm{C}$ compondo um arranjo fatorial $3 \times 4$. A contagem de germinação foi diária e consideradas germinadas na avaliação final as sementes que apresentaram protrusão da raiz primária maior ou igual a dois milímetros de comprimento. Com os resultados da emergência diária de plântas foi calculado o índice de velocidade de emergência (IVE), de acordo com Maguire (1962) e ao final do experimento, foram avaliadas as porcentagens de plântulas normais (Mapa, 1992). O delineamento experimental utilizado foi o inteiramente casualizado. As médias obtidas foram comparadas entre si pelo teste de Tukey a 5\%, sendo os dados em porcentagem transformados para $\operatorname{arco}$ seno $(\mathrm{x} / 100)^{1 / 2}$. 


\section{Resultados e Discussão}

No estudo da embebição (Figura 1) é possível observar que a absorção de água pelas sementes de jabuticabeira se deu de maneira lenta, o que é justificado pelo alto teor de água nas sementes no início do processo, uma característica das sementes recalcitrantes. Esse alto teor de umidade das sementes exerce força contrária à entrada de água nas células (pressão hidrostática), o que restringe a embebição (Popinigs, 1985). Nas quatro primeiras horas ocorre um aumento gradativo do peso das sementes, seguido de estabilização até as sete horas, a partir da qual se dá novamente a retomada do processo de absorção que prossegue até as 72 horas, quando a semente apresenta um aumento de cerca de $13,5 \%$ em relação ao peso inicial.

O processo de embebição é um fenômeno que se caracteriza pelo movimento de água para o interior das células, ocorrendo em conseqüência à polaridade das moléculas de água, que lhe confere a propriedade de aderência às superfícies carregadas. Esse processo é de fundamental importância para a germinação das sementes, pois esta só ocorrerá quando a semente absorver água necessária para o desencadeamento dos processos metabólicos que culminarão no desenvolvimento do embrião. Uma deficiente absorção de água pode comprometer o funcionamento das atividades fisiológicas envolvidas na germinação (Bewley e Black, 1994; Raven, 2001). Entre as espécies ocorre comportamento diferenciado no que se refere à velocidade de absorção de água por suas sementes, o que caracteriza seu padrão de embebição e de germinação (Carvalho e Nakagawa, 2000).

Na Tabela 1 são apresentados os resultados referentes a porcentagem de germinação de sementes de Jabuticabeira em diferentes substratos e temperaturas. As sementes apresentaram maiores valores de germinação na temperatura de $30{ }^{\circ} \mathrm{C}$ e no substrato constituído pela mistura terra, areia

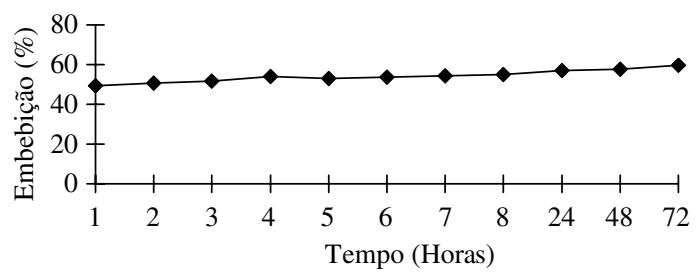

Figura 1. Curva de embebição de sementes de jabuticabeira (Myrciaria cauliflora Berg.) -CCA-UFES, Alegre-ES.
Tabela 1. Média da percentagem de germinação de sementes de jabuticabeira em diferentes substratos e temperaturas -

CCA-UFES, Alegre-ES

\begin{tabular}{lc}
\hline Substrato/Temperatura & Germinação (\%) \\
\hline Areia & $32,67 \mathrm{~A}$ \\
Terra+ areia+ esterco & $42,00 \mathrm{~A}$ \\
Pó de casca de coco & $4,67 \mathrm{~B}$ \\
Hortimix® & $5,67 \mathrm{~B}$ \\
$20{ }^{\circ} \mathrm{C}$ & \\
$30{ }^{\circ} \mathrm{C}$ & $15,75 \mathrm{~B}$ \\
$20-30{ }^{\circ} \mathrm{C}$ & $26,50 \mathrm{~A}$ \\
\hline
\end{tabular}

* Médias seguidas pela mesma letra não diferem estatisticamente pelo teste de Tukey com 5\% de probabilidade.

e esterco bovino $(\mathrm{T}+\mathrm{A}+\mathrm{E})$ em iguais proporções, sendo que estes fatores não interagiram significativamente. Tal resposta difere da obtida por Andrade e Martins (2003) que obtiveram resposta mais favorável em temperaturas mais baixas (15 e $20{ }^{\circ} \mathrm{C}$ ), nas quais chegaram a obter até $76 \%$ de germinação. A melhor temperatura para a germinação das sementes de jabuticabeira nas condições avaliadas foi $30^{\circ} \mathrm{C}$, podendo esta ser considerada a mais favorável, pois, segundo Alves et al. (2002), uma temperatura ótima é aquela na qual a maior porcentagem de germinação é obtida, dentro de um espaço mais curto de tempo. Além da temperatura, o substrato é outro fator importante no desencadeamento do processo germinativo. As características apresentadas pelo substrato atuam favorecendo ou prejudicando a germinação das sementes (Barbosa et al., 1985). Os maiores valores de germinação obtidos nos substratos areia pura (A) e $\mathrm{T}+\mathrm{A}+\mathrm{E}$, quando comparados aos substratos compostos por $\mathrm{PC}$ e $\mathrm{H}$, dos quais diferiram significativamente. Valores mais elevados de emergência foram obtidos por Alexandre et al. (2004) e Danner et al. (2007) que obtiveram valores de germinação de $77 \%$ e $80 \%$ respectivamente.

Em relação ao vigor, avaliado através ao índice de velocidade de emergência (Tabela 2 ) o maior valor foi obtido quando se utilizou o substrato $\mathrm{T}+\mathrm{A}+\mathrm{E}$ associado à temperatura de $30^{\circ} \mathrm{C}$. Esse resultado difere do obtido por Danner et al. (2007) que obtiveram maiores valores de IVE em substrato comercial. De modo geral, se observou que dentro de cada substrato, a temperatura de $30^{\circ} \mathrm{C}$ proporcionou os maiores valores de IVE, exceto quando se utilizou o substrato pó de casca de coco (PC), no qual não ocorreu diferença entre as temperaturas testadas. 
Também este substrato promoveu aos menores índices de germinação das sementes.

Constam na Tabela 3 as médias de massa fresca e massa seca de plantas de jabuticabeira. A maior média foi obtida no substrato composto por $\mathrm{T}+\mathrm{A}+\mathrm{E}$. Com relação à temperatura, resposta semelhante foi obtida para os dois parâmetros nas temperaturas de $20-30^{\circ}$ e $30^{\circ} \mathrm{C}$. Para Alexandre et al. (2004) ao comparar substrato comercial e substrato preparado com terra e vermicomposto, o segundo apresentou a vantagem de ser mais econômico, proporcionando boa formação de mudas devido, possivelmente, às suas características físicas e químicas, como maior porosidade o que favorece a retenção de água e aeração.

Quanto à formação de plantas normais (Tabela 4) independente do substrato utilizado, os melhores valores foram obtidos nas temperaturas de 20-30 e $30^{\circ} \mathrm{C}$. Os substratos que mais favoreceram a formação de plântulas normais foram $\mathrm{A} e \mathrm{~T}+\mathrm{A}+\mathrm{E}$, que não diferiram entre si.

Tabela 2. Índice de velocidade de emergência (IVE) de sementes de jabuticabeira em diferentes substratos e temperaturas - CCA-UFES, Alegre-ES

\begin{tabular}{llll}
\hline \multirow{2}{*}{ Substrato } & \multicolumn{3}{c}{ Temperatura } \\
\cline { 2 - 4 } & \multicolumn{1}{c}{$20{ }^{\circ} \mathrm{C}$} & $30{ }^{\circ} \mathrm{C}$ & $20-30{ }^{\circ} \mathrm{C}$ \\
\hline Areia & $0,086 \mathrm{Ab}$ & $0,28 \mathrm{Ba}$ & $0,19 \mathrm{ABab}$ \\
Terra+ areia+ esterco & $0,14 \mathrm{Ac}$ & $0,55 \mathrm{Aa}$ & $0,34 \mathrm{Ab}$ \\
Pó de casca de coco & $0 \mathrm{Aa}$ & $0,05 \mathrm{Ca}$ & $0,05 \mathrm{BCa}$ \\
Hortimix ${ }^{\circledR}$ & $0 \mathrm{Ab}$ & $0,14 \mathrm{BCa}$ & $0,03 \mathrm{Cab}$ \\
\hline
\end{tabular}

* Médias seguidas pela mesma letra, maiúsculas nas colunas e minúsculas nas linhas, não diferem estatisticamente pelo teste de Tukey com 5\% de probabilidade.
Tabela 3. Massa fresca e seca (mg.planta -1) de sementes de jabuticabeira em diferentes substratos e temperaturas - CCA-UFES, Alegre-ES

\begin{tabular}{lcc}
\hline Substrato/Temperatura & Massa fresca & Massa seca \\
\hline Areia & $72,82 \mathrm{AB}$ & $20,37 \mathrm{AB}$ \\
Terra+ areia+ esterco & $106,60 \mathrm{~A}$ & $25,71 \mathrm{~A}$ \\
Pó de casca de coco & $36,78 \mathrm{~B}$ & $10,77 \mathrm{~B}$ \\
Hortimix® & $59,90 \mathrm{~B}$ & $18,23 \mathrm{AB}$ \\
\hline $20^{\circ} \mathrm{C}$ & $17,56 \mathrm{~B}$ & $3,07 \mathrm{~B}$ \\
$30^{\circ} \mathrm{C}$ & $91,22 \mathrm{~A}$ & $26,70 \mathrm{~A}$ \\
$20-30{ }^{\circ} \mathrm{C}$ & $98,30 \mathrm{~A}$ & $26,54 \mathrm{~A}$ \\
\hline
\end{tabular}

* Médias seguidas pela mesma letra não diferem estatisticamente pelo teste de Tukey com $5 \%$ de probabilidade.

Tabela 4. Porcentagem de plantas normais de jabuticabeira germinadas em diferentes substratos e temperaturas - CCA-UFES, Alegre-ES

\begin{tabular}{lrrr}
\hline \multirow{2}{*}{ Substrato } & \multicolumn{3}{c}{ Temperatura } \\
\cline { 2 - 4 } & \multicolumn{1}{c}{$20{ }^{\circ} \mathrm{C}$} & \multicolumn{1}{c}{$30{ }^{\circ} \mathrm{C}$} & $20-30{ }^{\circ} \mathrm{C}$ \\
\hline Areia & $17,0 \mathrm{Ab}$ & $28,0 \mathrm{Aa}$ & $37,0 \mathrm{Aa}$ \\
Terra+ areia+ esterco & $17,0 \mathrm{Ab}$ & $37,0 \mathrm{Aa}$ & $50,0 \mathrm{Aa}$ \\
Pó de casca de coco & $0,0 \mathrm{Aa}$ & $4,0 \mathrm{Ba}$ & $11,0 \mathrm{Ba}$ \\
Hortimix@ & $0,0 \mathrm{Aa}$ & $9,0 \mathrm{Ba}$ & $5,0 \mathrm{Ba}$ \\
\hline
\end{tabular}

* Médias seguidas pela mesma letra, maiúsculas nas colunas e minúsculas nas linhas, não diferem estatisticamente pelo teste de Tukey com $5 \%$ de probabilidade.

\section{Conclusões}

A temperatura de $30^{\circ} \mathrm{C}$ foi a que mais favoreceu a germinação de sementes de jabuticabeira. Entre os substratos, àquele constituído pela mistura de terra, areia e esterco bovino em iguais proporções, possibilitou maior porcentagem de germinação e vigor de plântulas.

\section{Literatura Citada}

Albrecht, J.M.F.; Albuquerque, M.C.F.E. \& Silva, M.V.F.

1986 Influência da temperatura e do tipo de substrato na germinação de sementes de cerejeira. Rev. Brasileira de Sementes, Brasília, 8 (1): 49-55.

Andrade, R.A. De e Martins, A.B.G.

2003 Influence of the temperature in gernination of seeds of jabuticaba tree. Rev. Brasileira de Fruticultura. Jaboticabal, 25 (1): 197-198.

Alexandre, R.S.; Wagner Jr., A.; Negreiros, J.R. da S.; Lopes, J.C. e Dias, M.A.

2004 Efeito do estádio de maturação dos frutos e de substratos na germinação de sementes e desenvolvimento inicial de plântulas de jabuticabeira. In: Simpósio Nacional do
Morango, 2., Encontro de Pequenas Frutas e Frutas Nativas do Mercosul, 1., 2004, Pelotas. Resumos... Pelotas: Embrapa Clima Temperado, pp. 422-427.

Alexandre, R.S.; Wagner Jr., A.; Negreiros, J.R. da S.; Bruckner, C.H.; Lopes, J.C. e Dias, M.A.

2010. Seed germination and emergency of two myrciaria species in three substrates. Acta Horticulturae (ISHS). 864: 175-179.

Alves, E.D.; Paula, R.C.; Oliveira,A. P.; Bruno, R.L.A. e Diniz, A.A.

2002 Germinação de sementes de Minosa caesalpiniaefolia Benth. em diferentes substratos e temperaturas. Rev. Brasileira de Sementes, Brasília, 24 (1): 169-178. 
Barbosa, J.M., Barbosa, L.M., Silva, T.S. E Ferreira, D.T.L.

1985. Influência do substrato, da temperatura e do armazenamento sobre germinação de sementes de quatro espécies nativas. Ecossistema. Espírito Santo do Pinhal, 10 (1): 46-54.

Bewley, J.D.; Black, M.

1994 Seeds: physiology of development and germination. New York and London: Plenum Press. 445 p.

Borges, E.E.L. \& Rena, A.B.

1993 Germinação de sementes. In.: Aguiar, I.B.; Pina-Rodrigues, F.M.C. \& Figliolia, M.B. (coords.). Sementes Florestais Tropicais. Brasília: ABRATES. Cap. 3-6, pp. 83-136.

Carvalho, N.M. e Nakagawa, J.

2000 Sementes: ciência, tecnologia e produção. 4. ed. Jaboticabal: FUNEP, 588 p.

Danner, M.A.; Citadin, I.; Fernandes Junior, A.A.; Assmann,

A.P.; Mazaro, S.M.; Sasso; S.A.Z.

2007 Formação de mudas de jabuticabeira (Plinia sp.) em diferentes substratos e tamanhos de recipientes. Revista Brasileira Fruticultura. Jaboticabal. 29 (1): 179-182.

Donadio, L.C.

2005 Jabuticaba. Revista toda fruta, 2000. Disponivel em $<$ www.todafruta.com.br/todafruta/institucional $>$ acesso em: 20 de jan. 2005.

Emater-Ro (Associação de assistencia técnica e extensão rural do estado de Roraima)

2007 Jabuticaba. Disponível em: <http://www.emater-rondonia. com.br/Jabuticaba.htm > Acesso em: 03 de setembro de 2007.
Figliola, M.B., Oliveira, E.C. \& Piña-Rodrigues, F.C. 1993 Análise de sementes. In: Aguiar, I.B., Piña-Rodrigues, F.C.M. \& Figliola, M.B. (ed.). Sementes florestais tropicais. Brasília: Abrates. 173-174 p.

Gomes, R.P.

1987 Fruticultura brasileira.São Paulo: Nobel. 546 p.

Maguire, J.D.

1962 Speed of germination aid in selection and evaluation for seedling emergence and vigor. Crop science. Madison, 2: 176-177.

Manica, I.

2000 Frutas nativas, silvestres e exóticas 1: técnicas de produção e mercado: abiu, amora-preta, araçá, bacuri, biribá, carambola, cereja-do-rio-grande, jabuticaba. Porto Alegre: Cinco Continentes. 327 p.

Marcos-Filho, J.

1986 Germinação de sementes. In: Cícero, S.M.; Marcos-Filho, J. \& Silva, W.S. Atualização em produção de sementes. Piracicaba: Fundação Cargill, 11-39 p.

Ministério da Agricultura, Pecuária e Abastecimento, Brasil 1992 Regras para análises de sementes. Brasília: SNDA/ DNDV/CLAV. 365 p.

Popinigis, F. 1985 Fisiologia da semente. Brasília: AGIPLAN. 289 p.

Raven, P.H.

2001 Biologia Vegetal. Rio de Janeiro: Guanabara Koogan. $906 \mathrm{p}$. 
